\title{
Transfection of Neuroprogenitor Cells with Iron Nanoparticles for Magnetic Resonance Imaging Tracking: Cell Viability, Differentiation, and Intracellular Localization
}

\author{
Sosuke Miyoshi, PhD, ${ }^{1}$ Jennifer A. Flexman, BEng, ${ }^{1}$ Donna J. Cross, BSE, ${ }^{2,3}$ \\ Kenneth R. Maravilla, MD, ${ }^{4}$ Yongmin Kim, PhD, ${ }^{1,4}$ Yoshimi Anzai, MD, \\ Junko Oshima, MD, $\mathrm{PhD},{ }^{5}$ Satoshi Minoshima, MD, $\mathrm{PhD}^{1,2,3,4}$ \\ ${ }^{1}$ Department of Bioengineering, University of Washington, Seattle, WA, USA \\ ${ }^{2}$ Washington National Primate Research Center, University of Washington, 1959 N.E. Pacific Street, RR215 \\ Seattle, WA, 98195-7115, USA \\ ${ }^{3}$ Neuroscience Graduate Program, University of Michigan, Ann Arbor, MI, USA \\ ${ }^{4}$ Department of Radiology, University of Washington, Seattle, WA, USA \\ ${ }^{5}$ Department of Pathology, University of Washington, Seattle, WA, USA
}

\begin{abstract}
Purpose: Magnetic resonance imaging (MRI) can track labeled cells in the brain. The use of hemagglutinating virus of Japan envelopes (HVJ-Es) to effectively introduce the contrast agent to neural progenitor cells (NPCs) is limited to date despite their high NPC affinity.

Procedures: HVJ-Es and Lipofectamine 2000 were compared as transfection vehicles of superparamagnetic iron oxide (SPIO). Labeled NPCs were examined for iron content, MRI signal change, and fundamental cell characteristics. Prussian Blue staining was used after differentiation to determine SPIO localization.

Results: HVJ-Es transfected up to $12.5 \pm 8.8$ times more SPIO into NPCs. HVJ-Es do not affect cell viability or differentiation capability. Superparamagnetic iron oxide was disseminated in both the soma and neurites.

Conclusions: These findings indicate that HVJ-Es are an effective vehicle for SPIO transfection of NPCs. The intracellular localization after differentiation raises the question as to the capability of MRI to distinguish cell migration from axonal or dendritic growth in vivo.
\end{abstract}

Keywords: Cell transplantation, Pheochromocytoma cells (PC12), Neuronal regeneration, Magnetic resonance imaging, Superparamagnetic iron oxide, Cell tracking

\section{Introduction}

S tem cells are a promising source of neuroregeneration, and potential therapy applications are debated extensively. For in vivo applications of stem cells, the character-

Correspondence to: Satoshi Minoshima, MD, PhD; e-mail: minoshim@ u.washington.edu ization of cell behavior in its natural microenvironment is critical [1]. Traditionally, investigations have relied on tissue specimens to track cell activities, providing limited longitudinal information. To track cells in vivo, cells are labeled with an imaging contrast agent prior to implantation. Cells containing a magnetic label can be tracked noninvasively in the living brain using magnetic resonance imaging (MRI). This requires both efficient labeling and characterization before transplantation. 
Superparamagnetic iron oxide (SPIO) alters the MRI signal intensity. SPIO particles have been modified to increase their transfection into the cell $[2,3]$ and the functionality of the contrast agent $[2,4,5]$, such as integrating SPIO and fluorescence. However, these methods require complex procedures that are specific to the chemistry of the contrast agent, which precludes the general application of this technology to a diverse class of labeling materials. Cationic transfection agents have been used for magnetic labeling [6-8] but have also been associated with the formation of reactive oxygen intermediates and increased cell toxicity [9]. Moreover, cationic agents rely on the anionic property of the contrast agent for efficiency.

The paramyxovirus is an effective gene therapy vector for neural cells [10], and the reconstituted hemagglutinating virus of Japan envelope (HVJ-E) was initially investigated because of its unique iron oxide content [11]. The iron content of the native envelope, however, was undetectable by either atomic absorption spectrophotometry (AAS) or MRI in our laboratory (data unpublished). The HVJ-E viral vector was initially proposed by Ardizzoni et al. [12] as a transfection method for gold particles, subsequently tested with iron oxides by Hawrylak et al. [13], and used to label PC12 cells and astrocytes [14]. However, HVJ-E transfection of neural progenitor cells (NPCs) has not been characterized systematically in vitro.

The sialic acid residues on the abundant nerve growth factor (NGF) receptors of NPCs make HVJ-Es ideal candidates for contrast agent transfection because of their affinity for sialic acid [15]. We hypothesize that HVJ-Es will be an efficient way to transfect NPCs. Furthermore, HVJEs may be more effective for introducing larger particles and ensembles of particles and do not depend on the electrostatic characteristic of the contrast material, rendering the technique more versatile.

In this study, we investigated the effects of labeling on neural progenitor cell viability, differentiation capability, and intracellular localization of the contrast agent after differentiation, which has not been characterized previously. We quantified SPIO transfection by both HVJ-Es and Lipofectamine 2000, and we used this set of quantitative results to compare the two methods and determined if labeled NPCs retained their maturation capability and viability and to ensure suitability for transplantation.

\section{Materials and Methods}

\section{Cell Culture and Labeling}

Rat pheochromocytoma PC12 cells (ATCC, Manassas, VA) were used as a model for neuroprogenitor cells as these cells have been studied extensively for their differentiation capabilities as well as for potential therapeutic applications. PC12 cells were cultured on collagen-coated flask in RPMI 1640 medium supplemented with $10 \%$ heat-inactivated horse serum (HS), 5\% heat-inactivated fetal bovine serum (FBS), and $20 \mathrm{U} / \mathrm{ml}$ penicillin-streptomycin in a $5 \%$
$\mathrm{CO}_{2}$ air-humidified atmosphere at $37^{\circ} \mathrm{C}$. PC12 cells were labeled with Feridex (Feridex IV; Berlex Laboratories, Wayne, NJ) using either (1) inactivated HVJ-E vectors (GenomeOne, Ishihara Sangyo, Osaka, Japan), which retain the cell fusion ability of the native virus; or (2) a liposome agent, Lipofectamine 2000 (L2000; Invitrogen, Carlsbad, CA). HVJ-Es were used to encapsulate Feridex particles. Briefly, cells (approximately $10^{6} /$ well) grown in collagen-coated, 6-well plates were exposed to HVJ-Es containing Feridex at volumes of 3.2 or $10 \mu \mathrm{l}$ for six hours. Additionally, to increase transfection efficiency, plates were centrifuged $(3,000$ $\mathrm{rpm})$ at room temperature for 10 minutes. PC12 cells were also transfected with Feridex using L2000. After $10 \mu 1$ of L2000 was incubated with Feridex at volumes of 3.2 or $10 \mu \mathrm{l}$ at room temperature for 20 minutes, the L2000-Feridex complexes were then added to culture media and cells were cultured for six hours.

\section{Prussian Blue Staining}

Prussian Blue (PB) staining (Polysciences, Warrington, PA) was performed to visualize intracellular iron content and localization. After labeling, cells were fixed in $4 \%$ glutaraldehyde for 10 minutes and washed in phosphate-buffered saline (PBS). Staining took two changes of $2 \%$ potassium ferrocyanide in $2 \%$ hydrochloric acid solution at 10 minutes each. Cells were rinsed in PBS, and photographs were taken of representative fields using an upright, light microscope (Nikon Eclipse; Nikon, Tokyo, Japan) and $1360 \times 1036$ pixel, 24-bit, CCD digital camera (QImaging, Burnaby, BC, Canada). Eight images were taken with a $60 \times$ objective of each labeling method and volume of Feridex.

\section{Light Microscopy Image Analysis}

To quantitatively evaluate PB staining of transfected cells, observer-independent image analysis for light microscopy was developed. Microscopic red-green-blue (RGB) images of labeled cells after PB staining were analyzed using a custom software program. The PB-stained area and the cell area were both segmented from the background using automatic thresholding. Otsu's algorithm [16] is often used to automatically determine a threshold in bimodal images by minimizing intergroup variance. Otsu's algorithm was used to isolate the PB-stained area (SA) using the criteria of red chrominance (Eq. 1) and the cell area (CA) by using the criteria of overall intensity (Eq. 2).

$$
\begin{aligned}
& \text { Criteria }_{S A}=\frac{\text { Red }}{\text { Red }+ \text { Green }+ \text { Blue }} \\
& \text { Criteria }_{C A}=\frac{\text { Red }+ \text { Green }+ \text { Blue }}{3}
\end{aligned}
$$

Morphological opening and closing was performed on the region segmented as cell area to fill holes within the object. The staining percentage (SP) was calculated after segmentation using the SA and the CA:

$$
S P(\%)=\frac{S A}{C A} \times 100 \%
$$




\section{Atomic Absorption Spectrophotometry}

Atomic absorption spectrophotometry provides a quantitative measure of the iron content of a sample based on the atomic absorption of electromagnetic radiation. Atomic absorption spectrophotometry measurements served as a gold standard for the amount of SPIO transfection in this study. All cell samples were initially digested using an acid solution of $3: 1$ perchloric to nitric acid and heated for three hours at $60^{\circ} \mathrm{C}$. The iron content of labeled PC12 cells was determined using an atomic absorption spectrophotometer with an automatic pipetting system (PE-1500; Perkin-Elmer, Shelton, CT). A calibration curve was first established for various known concentrations of Feridex mixed with unlabeled PC12 cells to convert the relative values obtained for labeled cells to iron content. Samples of $10 \mu \mathrm{l}$ of cells were measured twice, and three independent samples of labeled cells for each labeling method and each concentration of Feridex were prepared. The concentration of cells was varied to ensure that the iron content was within the range of the calibration curve.

\section{Magnetic Resonance Imaging}

As SPIO particles affect the MRI signal intensity, samples of cells containing Feridex were imaged to provide estimates of the signal changes. To aim for future imaging in humans, we estimated signal changes using a clinically available MRI scanner with a pulse sequence that is also used routinely. Labeled cells $\left(10^{6}\right.$ cells/ $\mathrm{ml}$ ) suspended in $4 \% \mathrm{w} / \mathrm{w}$ gelatin in 5 -mm-diameter nuclear magnetic resonance (NMR) tubes were scanned with a 1.5-T MRI system (GE Signa; General Electric, Milwaukee, WI) using a custom-designed birdcage volume coil. A 3-D steady-state free precession (SSFP) pulse sequence was used with a TE and TR of 61 and 38 milliseconds, respectively, a flip angle of $30^{\circ}$, a field of view (FOV) of $6 \times 4 \mathrm{~cm}(256 \times 192$ square pixels), and a slice thickness of $2.0 \mathrm{~mm}$. Each scan consisted of the sample and a control of $10^{6}$ unlabeled cells $/ \mathrm{ml}$ suspended in $4 \% \mathrm{w} / \mathrm{w}$ gelatin. Region of interest (ROI) analysis was performed on the crosssectional view of the labeled cells at the slice corresponding to the middle of the coil with the best visible homogeneity to determine $\mathrm{T} 2 *$-weighted signal intensity averages for three samples each of both labeling methods and both volumes of Feridex. Each T2*weighted signal intensity average was normalized by dividing by the average signal intensity of the control in the same image to compensate for coil and scanner inhomogeneities.

\section{Cell Viability}

Proliferation of labeled cells was determined by a colorimetric assay using Cell Titer 96 Aqueous One Solution Cell Proliferation Assay Kit based on MTS tetrazolium ([3-(4,5-dimethyl-2-yl)-5-(3carboxymethoxyphenyl)-2-(4-sulfophenyl)-2 $H$-tetrazolium, inner salt]) and its formazan product (Promega, Madison, WI). The MTS assay provided information about mitochondrial respiratory chain activity and the mitochondrial oxidation state. PC12 cells were plated in collagen-coated 96 -well plates at $10^{4}$ cells in 100 $\mu \mathrm{l}$ assay solution per well. At selected times, $20 \mu \mathrm{l}$ MTS solution was added to each well and then incubated at $37^{\circ} \mathrm{C}$ for three hours. The absorbance of samples was measured at a wavelength of 490 nm using 96-well plate reader.

\section{Cell Susceptibility}

To assess the susceptibility to apoptosis in labeled cells, a cytotoxicity assay was performed. Hydrogen peroxide $\left(\mathrm{H}_{2} \mathrm{O}_{2}\right)$ is known to be toxic to nervous tissue and cells. Toxicity is not usually mediated by a direct effect of $\mathrm{H}_{2} \mathrm{O}_{2}$, but rather $\mathrm{H}_{2} \mathrm{O}_{2}$ is a precursor to the hydroxyl radical through its reaction with $\mathrm{Fe}^{2+}$ ions, resulting in DNA damage and possible cell death [17]. The LDH release assay was performed here to assess the effect of iron within the cell. Initially, cells (approximately $10^{4}$ cells/well) were plated on collagen-coated 96-well plates in $100 \mu$ l of culture medium. After overnight culture, medium was changed with 200 $\mu 1$ of low serum medium (RPMI 1640 supplemented with $0.5 \% \mathrm{HS}$ and $0.5 \%$ FCS) including a three-hour exposure to either 15 or 100 $\mu \mathrm{l}$ of the toxin $\mathrm{H}_{2} \mathrm{O}_{2}$. After treatment of the toxin, the supernatants were transferred into corresponding wells of an optically clear 96well plate. Reaction mixture was added to each well, and the wells were incubated for 30 minutes. The absorbance of the samples was measured at a wavelength of $490 \mathrm{~nm}$ using 96-well plate reader.

\section{Superparamagnetic Iron Oxide Removal by Cell Division}

We hypothesize that the reduction of the contrast agent over time can be attributed in part to dilution and/or removal during cell division, in addition to exocytosis through the ATP binding cassette $(\mathrm{ABC})$ transporter and lysosomal digestion. To address the importance of cell division in SPIO removal, cells were cultured either in a high or low serum environment after labeling with 10 $\mu 1$ of Feridex. PC12 cells were cultured on collagen-coated slide chamber in RPMI 1640 medium, either supplemented with (1) $10 \%$ HS, 5\% heat-inactivated FBS, and $20 \mathrm{U} / \mathrm{ml}$ penicillin-streptomycin (high serum); or (2) $0.5 \% \mathrm{HS}, 0.5 \% \mathrm{FBS}$, and $20 \mathrm{U} / \mathrm{ml}$ penicillin-streptomycin (low serum). All cells were cultured in a $5 \% \mathrm{CO}_{2}$ air-humidified atmosphere at $37^{\circ} \mathrm{C}$ for eight days. Cells in a high serum environment were expected to have a doubling period of 48 hours, and cells in a low serum environment were expected to remain dormant after the last phase of cycling. Prussian Blue staining and automated image analysis of five to seven microscopic images per group, with manual fine-tuning of the threshold to within $10 \%$ of the original value, was performed as previously described.

\section{Neuronal Differentiation}

To investigate intracellular localization of Feridex after neuronal differentiation, the differentiation of $\mathrm{PC} 12$ cells was induced by NGF. PC12 cells were plated onto collagen-coated 6-well plates or chamber slides at low density (approximately $10^{4}$ cells $/ \mathrm{cm}^{2}$ ) and allowed to attach to plates overnight in serum-containing medium. Cells were cultured under low serum medium (RPMI 1640 supplemented with $0.5 \%$ HS and $0.5 \%$ FCS) including $10 \mathrm{ng} / \mathrm{ml}$ NGF (Promega). Fresh NGF was added every second day with the medium change. Labeling with $10 \mu \mathrm{l}$ of Feridex was performed as previously described. After three days of NGF treatment, neurite outgrowth was measured using an inverted, light microscope (Nikon Diaphot 200; Nikon) and 1,300 × 1,030 pixel, 8-bit, CCD digital camera (Princeton Instruments, Trenton, NJ). Ten grayscale images yielding approximately 100 cells of two independent wells for each labeling characteristic (method and volume of Feridex) 
were used to measure the neurite outgrowth length and soma diameter using the Neuron Morpho plugin for ImageJ (National Institutes of Health, Bethesda, MD). The percentage of cells from each well with neurites longer than 1.5 times the diameter of the cell body was calculated from these measurements.

After seven days of culture with $10 \mathrm{ng} / \mathrm{ml} \mathrm{NGF}$ and after PB staining, representative fields of labeled, differentiated cells from three independent samples ( $\sim 100$ cells each) were photographed with $40 \times($ sample 1$)$ or $60 \times($ samples 2 and 3 ) objectives using the upright, light microscope and the 24-bit, digital camera. Redgreen-blue images were manually segmented for neurite outgrowth and soma areas and automatically segmented for PB-stained areas using Otsu's algorithm with manual fine-tuning of the threshold to within $10 \%$ of the original value. Dendritic and axon processes were grouped as neurite outgrowths as they were indistinguishable at this stage of differentiation under light microscopy. Segmented pixels representing PB staining were superimposed on the manually segmented cell areas to determine the percentage of PBstained area in the neurite outgrowth(s) with respect to total neurite outgrowth area $(A)$ as well as the percentage of PB-stained area in the soma with respect to total soma area $(B) . A$ and $B$ are computed as:

$$
\begin{aligned}
& A=\frac{(\# \text { of stained pixels in neurite outgrowths })}{(\text { total } \# \text { of pixels in neurite outgrowths })} \times 100 \% \\
& B=\frac{(\# \text { of stained pixels in soma })}{(\text { total } \# \text { of pixels in soma })} \times 100 \%
\end{aligned}
$$

\section{Statistical Analysis}

For analysis of the staining percentage using automated image analysis and iron content using atomic absorption spectrophotometry, statistical significance was determined using the Student's $t$ test for independent samples with $p<0.05$. For analysis of the T2*-weighted signal intensity, the MTS assay, the LDH assay, and neurite outgrowth significance was determined using ANOVA with $p<0.05$. Pearson's correlation constant was calculated between measurements with $p<0.05$. Significance for contrast localization in the neurite outgrowths or soma as a staining percentage was determined using one-way ANOVA with weighting for cell numbers per image $(p<0.05)$.

\section{Results}

\section{Cell Labeling and Prussian Blue Staining}

Prussian Blue staining revealed areas of the neuroprogenitor cells (rat pheochromocytoma PC12 cells) that contained iron (Fig. 1A-D) and provided an indication of the loading of the contrast agent after transfection that was quantified using image processing. Observer-independent segmentation of PB staining in light microscopy images showed a significantly greater labeling efficiency, by volume of Feridex, with HVJ-E transfection as compared to L2000 transfection (Fig. 1E). The average percentage of stained area to cell area of labeled cells using HVJ-E transfection was $27.64 \pm 3.76$ and $44.76 \pm 3.82 \%$ for 3.2 and $10 \mu$ of Feridex, respectively, and the average percentage of stained area to cell area of labeled cells using L2000 transfection was $19.17 \pm 2.51$ and $22.77 \pm 2.21 \%$ for 3.2 and $10 \mu \mathrm{l}$ of Feridex, respectively. There was significantly greater PB staining of cells transfected with HVJ-E as compared to L2000 for $3.2 \mu \mathrm{l}(p<0.01)$ and $10 \mu \mathrm{l}(p<0.01)$ of Feridex and between 3.2 and $10 \mu \mathrm{l}$ of Feridex for HVJ-E transfection $(p<0.05)$ and L2000 transfection $(p<0.05)$. The correlation coefficient between the average percentage of PB staining of one sample and the average amount of iron per cell based on AAS of three samples is $0.88(p<$ 0.01 ) using a logarithmic relationship. After exposure to $10 \mu \mathrm{l}$ of Feridex for six hours, cells showed no uptake of Feridex through endocytosis (data not shown).
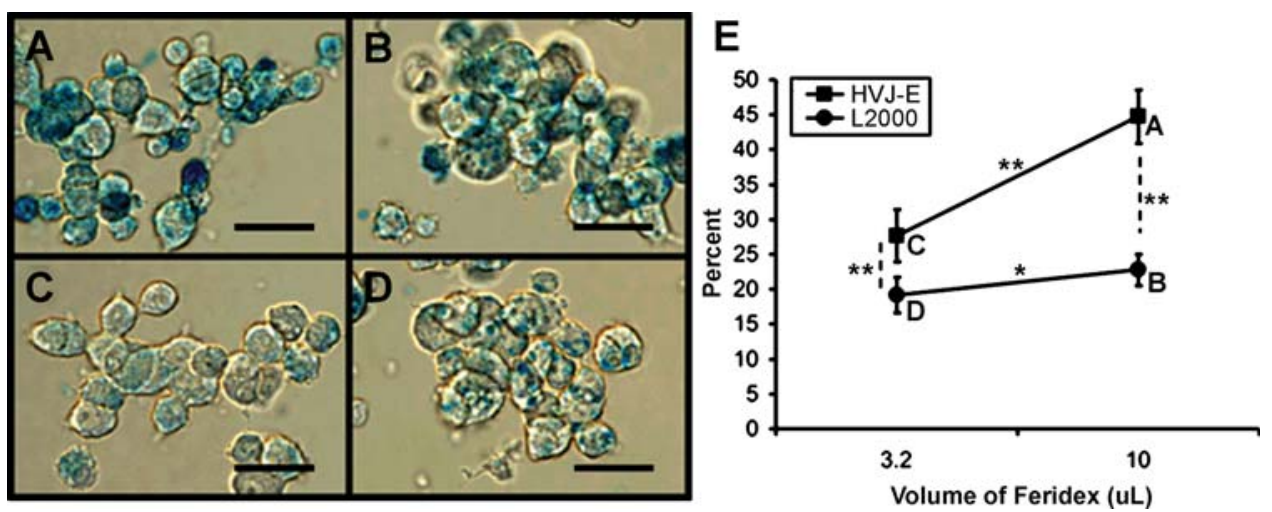

Fig. 1. Prussian Blue (PB) staining of transfected cells. Light microscopy photographs of PB-stained PC12 cells are shown using HVJ-E transfection with $10 \mu$ l of Feridex (A), L2000 transfection with $10 \mu$ of Feridex (B), HVJ-E transfection with $3.2 \mu$ l of Feridex (C), and L2000 transfection with $3.2 \mu$ of Feridex (D). Scale bars are $10 \mu \mathrm{m}$. Average percentages of PB-stained cell area to total cell area, by pixel, using two volumes of Feridex (3.2 and $10 \mu \mathrm{l})$ are shown and labeled A-D for HVJ-E and L2000 transfection methods (E). The PB-stained area of the image was automatically segmented from the rest of the image, as was the cell area. One star $\left(^{\star}\right)$ indicates significance at $p<0.05$, and two stars $\left(^{\star \star}\right)$ indicates significance at $p<0.01$. 


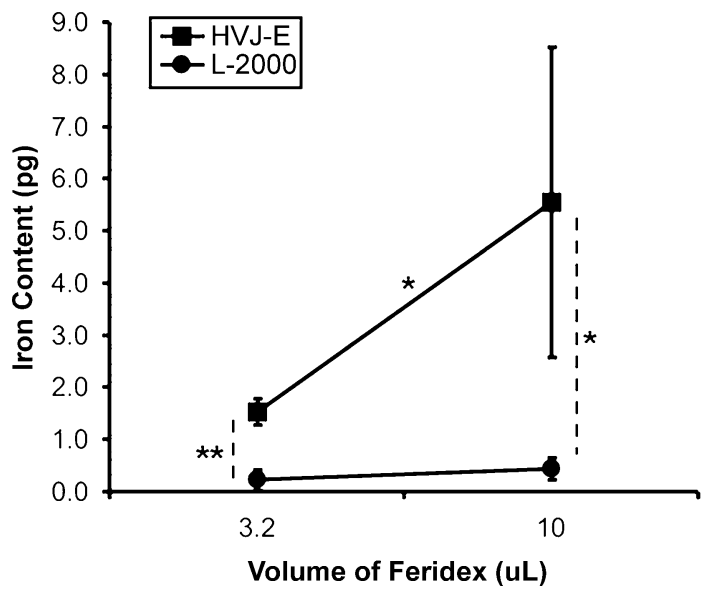

Fig. 2. Iron content per transfected cell using atomic absorption spectrophotometry (AAS). Average iron contents per cell based on AAS measurements for HVJ-E and L2000 transfection methods are shown using two volumes of Feridex $(3.2$ and $10 \mu \mathrm{l})$. Values were determined based on a calibration curve of known concentrations of Feridex mixed with unlabeled cells. One star $\left(^{*}\right)$ indicates significance at $p<0.05$, and two stars $\left({ }^{\star *}\right)$ indicates significance at $p<$ 0.01 .

\section{Atomic Absorption Spectrophotometry}

Atomic absorption spectrophotometry demonstrated that HVJ-E transfection resulted in a significantly greater iron content than L2000 transfection by volume of Feridex (Fig. 2). An optimal range of detectable iron concentrations (0.04-0.2 ppm) was used for the calibration curve of known concentrations of Feridex mixed with cells. The average iron content of cells labeled using HVJ-E transfection was $1.52 \pm 0.25$ and $5.55 \pm 2.97 \mathrm{pg} / \mathrm{cell}$ for 3.2 and $10 \mu \mathrm{l}$ of Feridex, respectively, and the iron content of cells labeled using L2000 transfection was $0.22 \pm 0.19$ and $0.44 \pm 0.21$ $\mathrm{pg} /$ cell for 3.2 and $10 \mu \mathrm{l}$ of Feridex, respectively. There was a significantly greater iron content of cells transfected with HVJ-E as compared to L2000 for $3.2 \mu \mathrm{l}(p<0.01)$ and $10 \mu \mathrm{l}(p<0.05)$ of Feridex and between 3.2 and $10 \mu \mathrm{l}$ of Feridex for HVJ-E transfection $(p<0.05)$.

\section{Magnetic Resonance Imaging}

Cells labeled with the Feridex reduced the T2*-weighted signal with MRI. For an equal volume of Feridex, the resulting change in MRI signal intensity was significantly greater with HVJ-E transfection as compared to L2000 transfection ( $p<0.01$, Fig. 3A). The average ratio of MRI T2*-weighted signal intensity of cells labeled using HVJ-E transfection to unlabeled cells was $0.322 \pm 0.058$ and 0.125 \pm 0.028 for 3.2 and $10 \mu \mathrm{l}$ of Feridex, respectively, and the average ratio of MRI T2*-weighted signal intensity of cells labeled using L2000 transfection to unlabeled cells was $0.637 \pm 0.040$ and $0.453 \pm 0.033$ for 3.2 and $10 \mu \mathrm{l}$ of Feridex, respectively. A standard curve was established using known concentrations of Feridex mixed with unlabeled cells and suspended in gelatin (Fig. 3B). From this curve, the amount of iron per cell was estimated to be $1.63 \pm 0.50$ and $5.77 \pm 0.96 \mathrm{pg} / \mathrm{cell}$ for 3.2 and $10 \mu \mathrm{l}$ of Feridex, respectively, using HVJ-E transfection and $0.22 \pm$ 0.05 and $0.71 \pm 0.13 \mathrm{pg} / \mathrm{cell}$ for 3.2 and $10 \mu \mathrm{l}$ of Feridex, respectively, using L2000 transfection. The linear correlation coefficient between the average iron content per cell predicted by MRI using the standard curve and the average amount of iron per cell based on AAS (gold standard) was $0.999(p<0.01)$ within the range of the current transfection efficiency despite a nonlinear relationship between $\mathrm{T} 2 *$-weighted signal intensity and the exact $\mathrm{T} 2$ relaxation value.
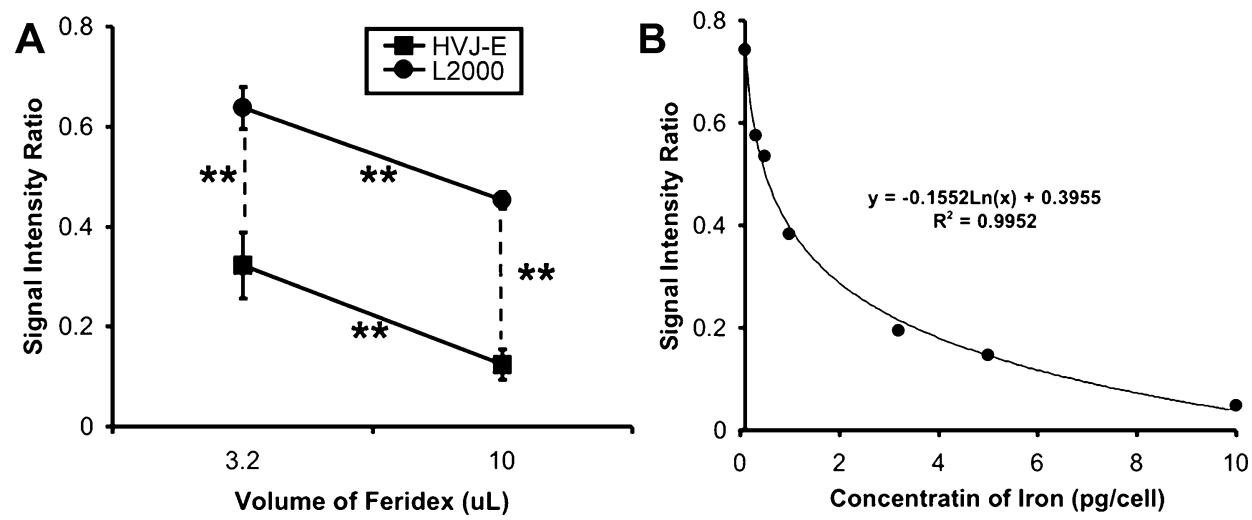

Fig. 3. Magnetic resonance imaging (MRI) signal intensity of transfected cells. MRI T2-weighted signal intensity changes are shown for cells in gelatin suspension in nuclear magnetic resonance tubes labeled using HVJ-E or L2000 transfection method and one of two volumes of Feridex (3.2 and $10 \mu \mathrm{l})(\mathbf{A})$. Intensity values are shown as a ratio between the signal intensity of the labeled cell suspension and the signal intensity of unlabeled control cell suspension in the same field of view and crosssection. A lower value represents a greater change in signal intensity. Two stars $\left(^{\star \star}\right)$ indicates significance at $p<0.01$. The standard curve for iron content based on MRI signal intensity is shown (B). 


\section{Cell Viability}

The MTS assay showed that cell viability was not affected by Feridex transfection using either HVJ-Es or L2000. This was evident from the quantitative analysis of the absorbance of three independent samples as a percentage of the absorbance of the control, unlabeled cells. The percentage of absorbance of cells labeled using HVJ-E transfection of unlabeled, control cells was $99.38 \pm 14.44$ and $99.00 \pm 11.71 \%$ for 3.2 and $10 \mu \mathrm{l}$ of Feridex, respectively. The percentage of absorbance of cells labeled using L2000 transfection of unlabeled, control cells was $101.20 \pm 11.56$ and $99.38 \pm$ $13.43 \%$ for 3.2 and $10 \mu \mathrm{l}$ of Feridex, respectively. There was no significant difference between the absorbance of both methods and the control (no transfection method) at any volume of Feridex. There was no significant difference between methods at any volume of Feridex.

\section{Cell Susceptibility}

As shown in Fig. 4, the transfection of cells with Feridex using either L2000 or HVJ-Es did not increase cell susceptibility to apoptosis as compared to unlabeled, control cells at two different levels of toxin: 15 and $100 \mu \mathrm{M}$ of $\mathrm{H}_{2} \mathrm{O}_{2}$. This was evident from the quantitative analysis of the percentage of LDH released at each level of added toxin from three independent samples. For $\mathrm{H}_{2} \mathrm{O}_{2}=100 \mu \mathrm{M}$, the percentage of LDH released of cells labeled using HVJ-E transfection was $55.96 \pm 7.02$ and $52.19 \pm 8.67 \%$ for 3.2 and $10 \mu \mathrm{l}$ of Feridex, respectively, and the percentage of LDH released of cells labeled using L2000 transfection was $57.66 \pm 5.13$ and $58.03 \pm 16.85 \%$ for 3.2 and $10 \mu \mathrm{l}$ of Feridex, respectively, whereas the control value was $56.53 \pm$ 9.17\%. For $\mathrm{H}_{2} \mathrm{O}_{2}=15 \mu \mathrm{M}$, the percentage of $\mathrm{LDH}$ released of cells labeled using HVJ-E transfection was $13.69 \pm 5.53$ and $16.11 \pm 1.64 \%$ for 3.2 and $10 \mu \mathrm{l}$ of Feridex, respectively, and the percentage of LDH released of cells labeled using L2000 transfection was $13.67 \pm 3.32$ and
$13.37 \pm 4.43 \%$ for 3.2 and $10 \mu \mathrm{l}$ of Feridex, respectively, whereas the control value was $13.45 \pm 2.05 \%$. There was no significant difference between the LDH release of both methods and the control (no transfection method) at any volume of Feridex. There was no significant difference between methods at any volume of Feridex.

\section{Superparamagnetic Iron Oxide Removal by Cell Division}

Cell division plays an important role in SPIO removal over time, as demonstrated by PB staining of cells cultured in high and low serum environments (Fig. 5A-D). Cells cultured in a high serum environment had lower Feridex content than cells cultured in a low serum environment for either L2000 or HVJ-E transfection, as demonstrated by quantitative image analysis of microscopic images of PBstained cells (Fig. 5E). The average percentage of stained area to cell area of labeled cells using HVJ-E transfection was $29.01 \pm 3.95$ and $10.17 \pm 1.68 \%$ for a low and high serum environment, respectively, and the average percentage of stained area to cell area of labeled cells using L2000 transfection was $21.18 \pm 5.38$ and $11.54 \pm 1.30 \%$ for a low and high serum environment, respectively. For the low serum environment, there was significantly more PB staining for cells transfected with HVJ-E as compared to L2000 $(p<0.01)$. For both transfection methods, there was significantly more PB staining for cells cultured in a low serum environment as compared to a high serum environment $(p<0.01)$.

\section{Neuronal Differentiation}

Cell differentiation was not affected by Feridex transfection using either HVJ-Es or L2000 (Fig. 6). This was demonstrated by the quantitative analysis of the percentage of cells with a ratio of the size of neurite outgrowth to soma

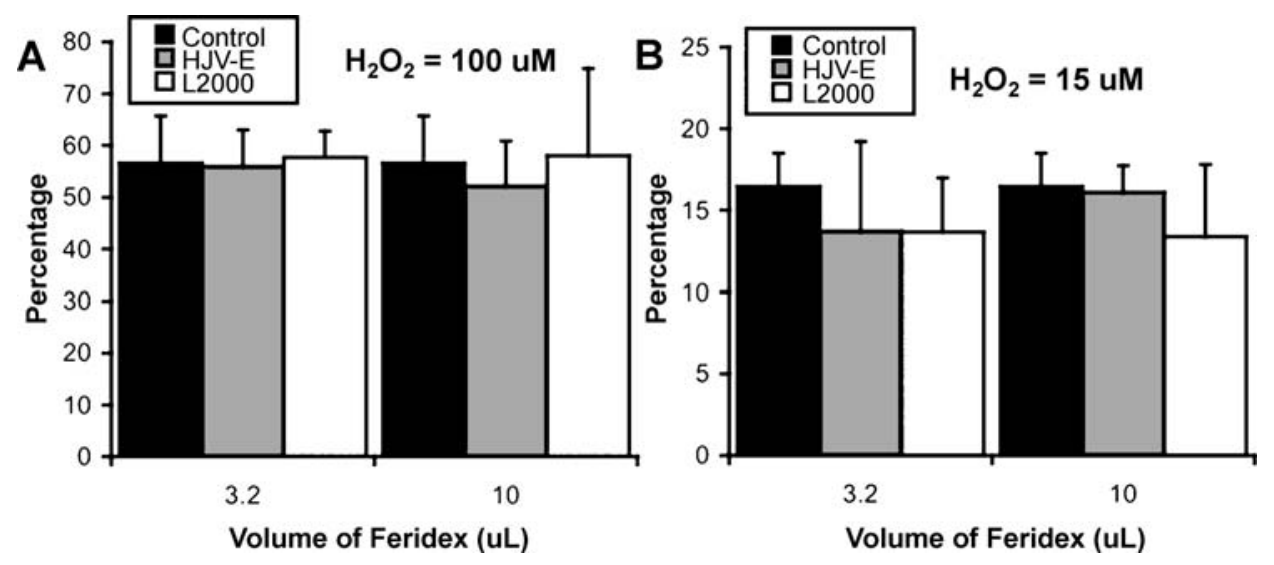

Fig. 4. Cell susceptibility after transfection with Feridex. Cell cytotoxicity, as determined using the LDH assay, is represented as the percentage of LDH release for HVJ-E and L2000 transfection methods using two volumes of Feridex (3.2 and $10 \mu \mathrm{l})$ as well as unlabeled, control cells. Values are shown for a toxin $\left(\mathrm{H}_{2} \mathrm{O}_{2}\right)$ concentration of $100 \mu \mathrm{M}(\mathbf{A})$ and $15 \mu \mathrm{M}(\mathbf{B})$. 

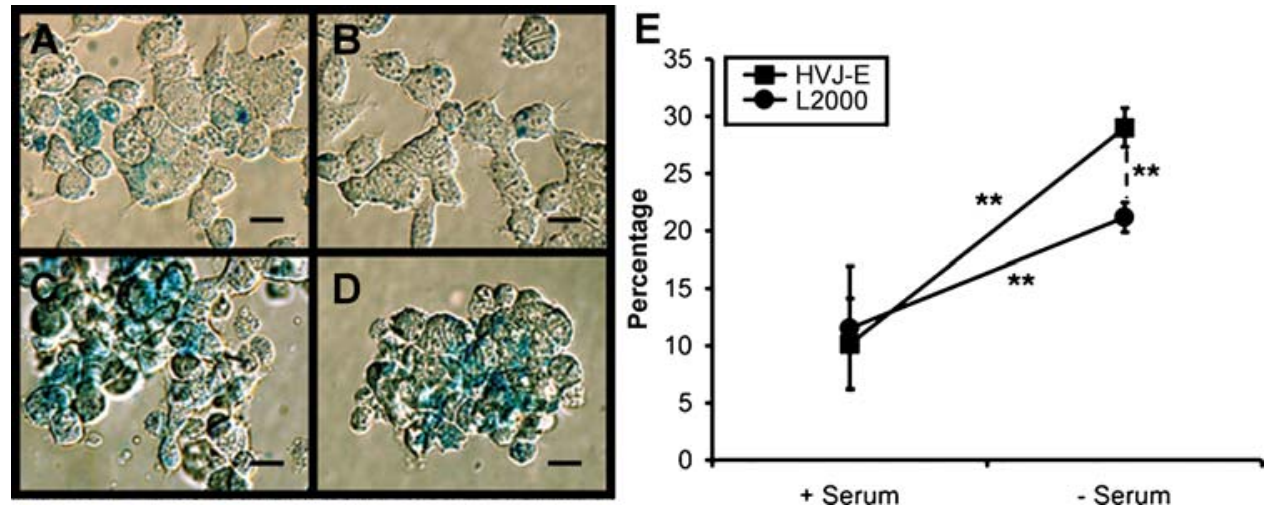

Fig. 5. Superparamagnetic iron oxide (SPIO) removal by cell division. Light microscopy photographs of Prussian-Bluestained (PB-stained) PC12 cells are shown after HVJ-E transfection and culture in a high serum environment (A), L2000 transfection in a high serum environment (B), HVJ-E transfection in a low serum environment (C), and L2000 transfection in a low serum environment (D). Scale bars are $10 \mu \mathrm{m}$. Average percentages of PB-stained cell area to total cell area, by pixel, are shown using different culture environments for HVJ-E and L2000 transfection methods (E). The PB-stained area of the image was automatically segmented from the rest of the image, as was the cell area. Two stars ${\left({ }^{\star \star}\right)}$ indicates significance at $p<0.01$.

diameter greater than 1.5. The percentage of differentiated cells, by this criterion, was $71.5 \pm 3.1,81.0 \pm 3.3$, and $67.7 \pm$ $4.2 \%$ for no transfection method, HVJ-E transfection, and L2000 transfection, respectively, when no Feridex was added. The percentage of differentiated cells was $70.4 \pm$ 4.2 and $67.6 \pm 1.4 \%$ for HVJ-E and L2000 transfection, respectively, when $3.2 \mu \mathrm{l}$ of Feridex was added. The percentage of differentiated cells was $70.1 \pm 6.5$ and 75.0 $\pm 4.8 \%$ for HVJ-E and L2000 transfection, respectively, when $10 \mu \mathrm{l}$ of Feridex was added. There was no significant difference between both methods and the control (no transfection method) at any volume of Feridex. There was

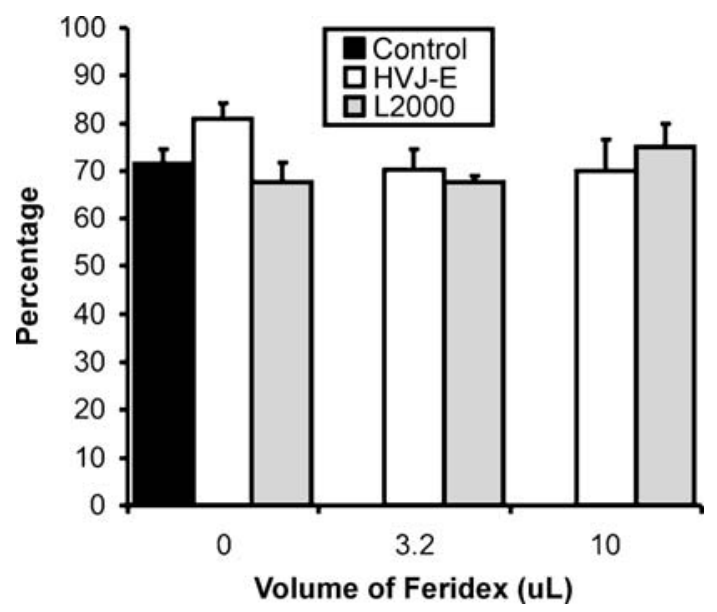

Fig. 6. Cell differentiation after transfection with Feridex. Percentages of differentiated cells are shown after nerve growth factor (NGF) treatment for HVJ-E and L2000 transfection methods using three volumes of Feridex $(0,3.2$, and $10 \mu \mathrm{l})$ as well as unlabeled, control cells. Differentiated cells are those that have neurite outgrowth length greater than $1.5 \times$ the soma diameter. no significant difference between methods at any volume of Feridex.

\section{Intracellular Localization of Superparamagnetic Iron Oxide}

Feridex was localized in both the neurite outgrowths and the soma of mature neurons, differentiated after labeling with $10 \mu$ of Feridex over 7 days with NGF, but more abundant in the soma (Fig. 7A). There was significantly more PB staining in both the soma and the neurite outgrowths for HVJ-E transfection as compared to L2000 transfection over three samples ( $p<0.05$, Fig. 7B). The average percentage of stained area to cell area of labeled cells using HVJ-E transfection was $8.06 \pm 2.15$ and $3.13 \pm 0.60 \%$ for the soma and neurite outgrowths, respectively, and the average percentage of stained area to cell area of labeled cells using L2000 transfection was $4.04 \pm 1.32$ and $1.76 \pm 0.24 \%$ for the soma and neurite outgrowths, respectively. For both transfection methods, there was significantly more PB staining in the soma as compared to the neurite outgrowths $(p<0.05)$.

\section{Discussion}

In this study, we investigated the use of a viral vector, HVJ-E, to efficiently deliver SPIO-containing Feridex to neural progenitor cells and compared it to a known charge-based transfection method, L2000, for (1) transfection efficiency, based on PB staining, iron content, and T2*-weighted MRI signal intensity; (2) viability; (3) susceptibility; and (4) differentiation, based on contrast material localization and neurite outgrowth. We showed that HVJ-E could transfect efficiently neuroprogenitor cells that are known to have abundant SA residues and that no significant toxicity, 

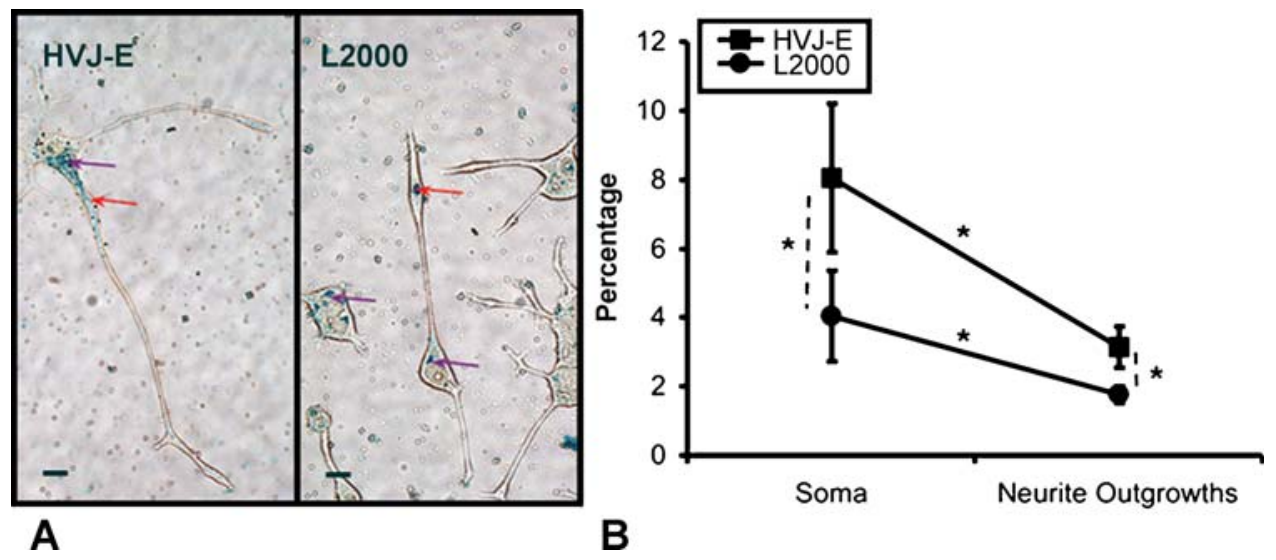

Fig. 7. Intracellular localization of Feridex after differentiation. Light microscopy photographs of Prussian-Blue-stained (PBstained) PC12 cells are shown for HVJ-E and L2000 transfection methods and $10 \mu \mathrm{l}$ of Feridex after nerve growth factor (NGF)induced differentiation (A). Scale bars are $10 \mu \mathrm{m}$, and red and purple arrows indicate staining in the neurite outgrowths and soma, respectively. Average percentage of PB-stained cell area to total cell area, by pixel, is shown for the neurite outgrowths and the soma for HVJ-E and L2000 transfection methods and $10 \mu \mathrm{l}$ of Feridex after NGF-induced differentiation (B). One star $\left(^{*}\right)$ indicates significance at $p<0.05$.

cell susceptibility, or impaired differentiation capacity was found. After 7 days of differentiation, cells transfected with HVJ-Es contained a significantly greater amount of iron, a characteristic that is essential to longitudinal MRI cell tracking. One of the important observations was that, after differentiation, Feridex was present in both the soma and growing axons and dendrites of mature neurons.

We chose to use a viral vector delivery system based on HVJ-E for SPIO partly because of the great interest in using HVJ-E as a tool for gene therapy. HVJ-E's high affinity for cells in the central nervous system and its low level of toxicity render it a promising choice for human trials [10]. Furthermore, the fact that this delivery technique is based on an encapsulation process rather than electrostatic attraction makes it a versatile alternative for the transfection of a wide range of contrast agents. Other agents commonly used in gene transfection, such as L2000 and poly-L-lysine, depend on the anionic charge of the contrast agent and may not be effective if chemical modifications are made to SPIO prior to transfection.

In this analysis, we demonstrated by using the paramyxoviral envelope HVJ-E that the contrast material Feridex was introduced more efficiently through the cell membrane as compared to the liposomal reagent L2000. This efficacy was clearly indicated by three different independent assays, namely, PB staining, AAS, and T2*weighted MRI. As shown in Fig. 2, the amount of staining for iron in cells transfected using HVJ-E is significantly greater for both volumes of Feridex. This trend is shown again in Fig. 3, with the average iron content per cell. There was a good correlation between the area percentage of $\mathrm{PB}$ staining and the iron content measured by AAS, indicating that image processing of stained cells represents a reasonable tool for evaluating transfection efficiency for quality control that is both inexpensive and accessible.
The difference in iron content of cells transfected using HVJ-E for an increasing volume of Feridex was greater than that determined for transfection with L2000. This may indicate that HVJ-E represents a more versatile method of introducing a greater amount of Feridex into the cell. The difference in PB-stained area and iron content between the two volumes of Feridex using L2000 was much smaller and could indicate that L2000 has a lower saturation point for the amount of SPIO or is inconsistent in its efficiency.

Magnetic resonance imaging of labeled cells in gelatin suspension, as shown in Fig. 3, demonstrated that transfection with HVJ-E had a greater effect on MRI signal intensity than transfection with L2000 given the same amount of Feridex. We can predict with reasonable accuracy the iron content of labeled cells in suspension based on an MRI signal intensity curve of iron standards, where the signal intensity ratio of labeled cells to unlabeled cells is linear with the $\log$ of actual iron content. The predicted values of iron content can be directly compared to those obtained through AAS with significant correlation. This suggests that the simple T2*-weighted MRI signal intensity of labeled cells is predictive of iron content within the range of the current transfection efficiency. However, an extrapolation to in vivo measurements may require a quantitative measurement of relaxation time for better estimates of SPIO content and requires further testing.

It is important to quantitatively assess the susceptibility to apoptosis of labeled cells because of the many immediate stresses that cells may encounter after transplantation into tissue, such as oxidative, radical formation, inflammatory, and physical. Moreover, the $\mathrm{Fe}^{2+}$ ion can react with hydrogen peroxide, $\mathrm{H}_{2} \mathrm{O}_{2}$, to produce the hydroxyl radical, which has been known to cause tissue and DNA damage [17]. The MTS assay demonstrated that cell labeling with Feridex using either HVJ-E or L2000 transfection had no significant 
effect on cell viability. Labeling with Feridex has not been shown to affect cell viability with either method of transfection [6, 13]. The LDH assay showed that there was no increase in susceptibility to apoptosis in the presence of $\mathrm{H}_{2} \mathrm{O}_{2}$, even at high concentrations, after labeling with either method of transfection. Whereas previous studies have shown that L2000 is associated with the formation of free radicals, for the amount of liposomal reagent used here, the toxic effect was not significant [9]. However, to increase the transfection efficiency to the level seen by HVJ-E transfection, an increase in the amount of L2000 will be required, which may in turn increase the cell's susceptibility to toxicity.

Increased retention of the magnetic label in transplanted cells enables cell tracking over a longer period of time. The contrast agent is diluted and/or removed during each cell cycle, which reduces the longevity of the detectable MRI signal. After 8 days in high serum-containing medium, the iron content, as determined with PB staining, was significantly lower than the iron content of dormant cells. This result supports our hypothesis that cell cycling plays a critical role in the reduction of the ability of MRI to detect labeled cells over time. However, the nonlinear relationship between PB staining and very low iron content as measured by AAS, in addition to an inexact measure of cell cycle arrest under low serum conditions, prevents the quantification of iron loss because of cell cycling. The lack of significant difference between the percentage of PB staining of each method in high serum-containing medium is interesting in that it may indicate that, despite the higher initial iron content of cells transfected with HVJ-E, as demonstrated in Fig. 1, the iron content after approximately a few cell cycles is similar relative to that of cells transfected with L2000. It is possible that the transfection method influences the rate of removal of the contrast agent during cycling, such as through a packing mechanism, but requires further investigation.

PC12 cells represent a well-established neural progenitor model for examining the effect of neuronal differentiation using NGF. Nerve growth factor drives the differentiation of PC12 cells into cells that closely resemble sympathetic neurons in vitro. Using this neuronal differentiation model, we have investigated the differentiation potential of labeled cells in vivo. The percentage of differentiated cells after labeling using either method of transfection was not significantly different from the percentage of differentiated, unlabeled cells. Neither the two transfection methods presented here, nor the presence of Feridex within the cell, adversely affected the maturation capability of PC12 cells, and these facts help establish suitability for transplantation.

Neural progenitor cells are multipotent and can differentiate into neurons, astrocytes, and oligodendrocytes both in vitro and in vivo. As differentiation is accompanied by dramatic morphological changes after maturation, it is very important to determine the intracellular localization of SPIO particles. This information will be useful for interpreting
MR images of labeled cells in vivo. After 7 days of differentiation, according to PB staining, Feridex is located in the soma and neurite outgrowths of neurons but remains more abundant in the soma. There is significantly more PB staining in both the soma and neurite outgrowths of cells transfected with HVJ-Es as compared to cells transfected with L2000. This fact may reiterate the initial increase in transfection efficiency for HVJ-E or suggest that the method of contrast agent encapsulation within the cytoplasm of the HVJ-E transfection method can resist certain mechanisms of contrast removal during maturation in the absence of cell cycling, such as exocytosis and digestion. The presence of iron in cells after differentiation is extremely important for performing cell tracking over extended periods. The presence of contrast agent within the axons and dendrites of mature cells poses a new question as to the distinction between cell migration and the growth of axons and dendrites in vivo and may necessitate the development of a mechanism whereby the contrast agent is fixed selectively into one intracellular location.

Of biological interest are the subcellular mechanisms by which Feridex particles are shuttled out to the axons. Billotey et al. [18] showed, using transmission electron microscopy (TEM), that within murine macrophages, ironcontaining, anionic maghemite nanoparticles were incorporated into the cell through the endocytotic pathway after incubation. Within fetal neuronal cell grafts, Kott et al. [19] demonstrated, using TEM, that gold nanoparticles of $10-200 \mathrm{~nm}$ in size were associated with many cytoplasmic membranes and even the nuclear compartment after transfection with reconstituted HVJ-Es, and particles were located within the axons and dendrites after maturation in vivo. It is likely that the method of transfection, such as viral or endosomal, plays a significant role in the mechanism of intracellular particle movement. Superparamagnetic iron oxide particles introduced within the cell using HVJ-E, which releases its contents directly into the cytoplasm, may have a greater ability to migrate within the cell than particles contained within endosomes or lysosomes given that HVJ-E transfection has resulted in nanoparticles within the nucleus as well as associated with membranes. This, in turn, may facilitate transport of the contrast agent to the axons and/or dendrites during and after differentiation.

\section{Conclusion}

Effective labeling methods encompass a balance between introducing a large amount of SPIOs into the cell and the consequential cytotoxicity associated with the presence of SPIOs and the transfection method. We have demonstrated here an intracellular paramagnetic labeling method that takes the considerations of not only iron content within the cell but also the proliferation, susceptibility to apoptosis, and distribution of the contrast agent after differentiation of labeled cells. The increased efficiency of HVJ-E transfec- 
tion is important to extend the window of detection for in vivo MRI imaging of implanted cells. The distribution of contrast material within the axons and dendrites after maturation of neural progenitor cells opens the door for specifically targeted contrast enhancement at a molecular level and the potential to visualize axonal growth for neuroregeneration, which requires further investigation.

Acknowledgements. This work was supported by NIH/NCRR P51 RR000166 and in part by funding from the Natural Science and Engineering Research Council of Canada. The authors would like to thank Berlex Laboratories (Wayne, NJ) for their donation of Feridex IV and Raja Atallah from the Environmental Health Laboratory for his help with the atomic absorption spectrophotometry.

\section{References}

1. Le Belle JE, Svendsen CN (2002) Stem cells for neurodegenerative disorders: Where can we go from here? BioDrugs 16:389-401

2. Lewin M, Carlesso N, Tung CH, et al. (2000) Tat peptide-derivatized magnetic nanoparticles allow in vivo tracking and recovery of progenitor cells. Nat Biotechnol 18:410-414

3. Bulte JW, Douglas T, Witwer B, et al. (2001) Magnetodendrimers allow endosomal magnetic labeling and in vivo tracking of stem cells. Nat Biotechnol 19:1141-1147

4. Hinds KA, Hill JM, Shapiro EM, et al. (2003) Highly efficient endosomal labeling of progenitor and stem cells with large magnetic particles allows magnetic resonance imaging of single cells. Blood 102:867-872

5. Modo M, Cash D, Mellodew K, et al. (2002) Tracking transplanted stem cell migration using bifunctional, contrast agent-enhanced, magnetic resonance imaging. NeuroImage 17:803-811

6. Van den Bos EJ, Wagner A, Mahrholdt H, et al. (2003) Improved efficacy of stem cell labeling for magnetic resonance imaging studies by the use of cationic liposomes. Cell Transplant 12:743-756
7. Arbab AS, Bashaw LA, Miller BR, et al. (2003) Intracytoplasmic tagging of cells with ferumoxides and transfection agent for cellular magnetic resonance imaging after cell transplantation: Methods and techniques. Transplantation 76:1123-1130

8. Hoehn M, Kustermann E, Blunk J, et al. (2002) Monitoring of implanted stem cell migration in vivo: A highly resolved in vivo magnetic resonance imaging investigation of experimental stroke in rat. Proc Natl Acad Sci U S A 99:16267-16272

9. Dokka S, Toledo D, Shi X, et al. (2000) Oxygen radical-mediated pulmonary toxicity induced by some cationic liposomes. Pharm Res 17:521-525

10. Shimamura M, Morishita R, Endoh M, et al. (2003) HVJ-envelope vector for gene transfer into central nervous system. Biochem Biophys Res Commun 300:464-471

11. Popa LM, Repanovici R, Mihalache O, et al. (1982) EPR and Mossbauer spectroscopy investigations on the metal ion contents of Sedai virus components. Virologie 33:271-278

12. Ardizzoni SC, Michaels A, Arendash GW (1988) Labeling of neural cells by gold-filled Sendai virus envelopes before intracerebral transplantation. Science 239:635-637

13. Hawrylak N, Ghosh P, Broadus J, et al. (1993) Nuclear magnetic resonance (NMR) imaging of iron oxide-labeled neural transplants. Exp Neurol 121:181-192

14. Toyoda K, Tooyama I, Kato M, et al. (2004) Effective magnetic labeling of transplanted cells with HVJ-E for magnetic resonance imaging. NeuroReport 15:589-593

15. Vale RD, Hosang M, Shooter EM (1985) Sialic acid residues on NGF receptors on PC12 cells. Dev Neurosci 7:55-64

16. Otsu N (1979) A threshold selection method from gray-level histogram. IEEE Trans Syst Man Cybern 8:62-66

17. Halliwell B (1992) Reactive oxygen species and the central nervous system. J Neurochem 59:1609-1623

18. Billotey C, Wilhelm C, Devaud M, et al. (2003) Cell internalization of anionic maghemite nanoparticles: Quantitative effect on magnetic resonance imaging. Magn Reson Med 49:646-654

19. Kott JN, Westrum LE, Ardizzoni SC, et al. (1991) Ultrastructural localization of gold particles within neural grafts labeled with goldfilled Sendai viral envelopes. J Electron Microsc Tech 18:197-202 